\title{
Dynamics of a fourth-order system of rational difference equations
}

\section{Q Din ${ }^{*}$, MN Qureshi and A Qadeer Khan}

${ }^{*}$ Correspondence: qamar.sms@gmail.com University of Azad Jammu and Kasmir, Muzaffarabad, Pakistan

\begin{abstract}
In this paper, we study the equilibrium points, local asymptotic stability of an equilibrium point, instability of equilibrium points, periodicity behavior of positive solutions, and global character of an equilibrium point of a fourth-order system of rational difference equations of the form

$$
x_{n+1}=\frac{\alpha x_{n-3}}{\beta+\gamma y_{n} y_{n-1} y_{n-2} y_{n-3}}, \quad y_{n+1}=\frac{\alpha_{1} y_{n-3}}{\beta_{1}+\gamma_{1} x_{n} x_{n-1} x_{n-2} x_{n-3}}
$$

$n=0,1, \ldots$, where the parameters $\alpha, \beta, \gamma, \alpha_{1}, \beta_{1}, \gamma_{1}$ and initial conditions $x_{0}, x_{-1}, x_{-2}$,

$x_{-3}, y_{0}, y_{-1}, y_{-2}, y_{-3}$ are positive real numbers. Some numerical examples are given to verify our theoretical results.
\end{abstract}

MSC: 39A 10; 40A05

Keywords: system of rational difference equations; stability; global character

\section{Introduction and preliminaries}

The theory of discrete dynamical systems and difference equations developed greatly during the last twenty-five years of the twentieth century. Applications of difference equations also experienced enormous growth in many areas. Many applications of discrete dynamical systems and difference equations have appeared recently in the areas of biology, economics, physics, resource management, and others. The theory of difference equations occupies a central position in applicable analysis. There is no doubt that the theory of difference equations will continue to play an important role in mathematics as a whole. Nonlinear difference equations of order greater than one are of paramount importance in applications. Such equations also appear naturally as discrete analogues and as numerical solutions of differential and delay differential equations which model various diverse phenomena in biology, ecology, physiology, physics, engineering and economics. It is very interesting to investigate the behavior of solutions of a system of higher-order rational difference equations and to discuss the local asymptotic stability of their equilibrium points.

Cinar [1] investigated the periodicity of the positive solutions of the system of rational difference equations

$$
x_{n+1}=\frac{1}{y_{n}}, \quad y_{n+1}=\frac{y_{n}}{x_{n-1} y_{n-1}} .
$$

(C) 2012 Din et al.; licensee Springer. This is an Open Access article distributed under the terms of the Creative Commons Attribution License (http://creativecommons.org/licenses/by/2.0), which permits unrestricted use, distribution, and reproduction in any medium, provided the original work is properly cited. 
Stević [2] studied the system of two nonlinear difference equations

$$
x_{n+1}=\frac{u_{n}}{1+v_{n}}, \quad y_{n+1}=\frac{w_{n}}{1+s_{n}},
$$

where $u_{n}, v_{n}, w_{n}, s_{n}$ are some sequences $x_{n}$ or $y_{n}$.

Kurbanli [3] studied the behavior of positive solutions of the system of rational difference equations

$$
x_{n+1}=\frac{x_{n-1}}{y_{n} x_{n-1}-1}, \quad y_{n+1}=\frac{y_{n-1}}{x_{n} y_{n-1}-1}, \quad z_{n+1}=\frac{1}{y_{n} z_{n}} .
$$

Bajo and Liz [4] investigated the global behavior of the difference equation

$$
x_{n+1}=\frac{x_{n-1}}{a+b x_{n-1} x_{n}}
$$

for all values of real parameters $a, b$.

Kalabusiić, Kulenović, and Pilav [5] investigated the global dynamics of the following systems of difference equations:

$$
x_{n+1}=\frac{\alpha_{1}+\beta_{1} x_{n}}{A_{1}+y_{n}}, \quad y_{n+1}=\frac{\gamma_{2} y_{n}}{A_{2}+B_{2} x_{n}+y_{n}} .
$$

Kurbanli, Çinar, and Yalçinkaya [6] studied the behavior of positive solutions of the system of rational difference equations

$$
x_{n+1}=\frac{x_{n-1}}{y_{n} x_{n-1}+1}, \quad y_{n+1}=\frac{y_{n-1}}{x_{n} y_{n-1}+1} \text {. }
$$

Touafek and Elsayed [7] studied the periodic nature and got the form of the solutions of the following systems of rational difference equations:

$$
x_{n+1}=\frac{x_{n-3}}{ \pm 1 \pm x_{n-3} y_{n-1}}, \quad y_{n+1}=\frac{y_{n-3}}{ \pm 1 \pm y_{n-3} x_{n-1}} \text {. }
$$

Similarly, Touafek, and Elsayed [8] studied the periodicity nature of the following systems of rational difference equations:

$$
x_{n+1}=\frac{y_{n}}{x_{n-1}\left( \pm 1 \pm y_{n}\right)}, \quad y_{n+1}=\frac{x_{n}}{y_{n-1}\left( \pm 1 \pm x_{n}\right)} .
$$

Recently, Zhang, Yang, and Liu [9] studied the dynamics of a system of the rational thirdorder difference equation

$$
x_{n+1}=\frac{x_{n-2}}{B+y_{n} y_{n-1} y_{n-2}}, \quad y_{n+1}=\frac{y_{n-2}}{A+x_{n} x_{n-1} x_{n-2}}, \quad n=0,1, \ldots
$$

Our aim in this paper is to investigate the dynamics of a system of fourth-order rational difference equations

$$
x_{n+1}=\frac{\alpha x_{n-3}}{\beta+\gamma y_{n} y_{n-1} y_{n-2} y_{n-3}}, \quad y_{n+1}=\frac{\alpha_{1} y_{n-3}}{\beta_{1}+\gamma_{1} x_{n} x_{n-1} x_{n-2} x_{n-3}},
$$


$n=0,1, \ldots$, where the parameters $\alpha, \beta, \gamma, \alpha_{1}, \beta_{1}, \gamma_{1}$ and initial conditions $x_{0}, x_{-1}, x_{-2}, x_{-3}$, $y_{0}, y_{-1}, y_{-2}, y_{-3}$ are positive real numbers. This paper is a natural extension of $[9,10]$.

Let us consider an eight-dimensional discrete dynamical system of the form

$$
\begin{aligned}
& x_{n+1}=f\left(x_{n}, x_{n-1}, x_{n-2}, x_{n-3}, y_{n}, y_{n-1}, y_{n-2}, y_{n-3}\right), \\
& y_{n+1}=g\left(x_{n}, x_{n-1}, x_{n-2}, x_{n-3}, y_{n}, y_{n-1}, y_{n-2}, y_{n-3}\right),
\end{aligned}
$$

$n=0,1, \ldots$, where $f: I^{4} \times J^{4} \rightarrow I$ and $g: I^{4} \times J^{4} \rightarrow J$ are continuously differentiable functions and $I, J$ are some intervals of real numbers. Furthermore, a solution $\left\{\left(x_{n}, y_{n}\right)\right\}_{n=-3}^{\infty}$ of the system (1.2) is uniquely determined by initial conditions $\left(x_{i}, y_{i}\right) \in I \times J$ for $i \in$ $\{-3,-2,-1,0\}$. Along with the system (1.2), we consider the corresponding vector map $F=\left(f, x_{n}, x_{n-1}, x_{n-2}, x_{n-3}, g, y_{n}, y_{n-1}, y_{n-2}, y_{n-3}\right)$. An equilibrium point of (1.2) is a point $(\bar{x}, \bar{y})$ that satisfies

$$
\begin{aligned}
& \bar{x}=f(\bar{x}, \bar{x}, \bar{x}, \bar{x}, \bar{y}, \bar{y}, \bar{y}, \bar{y}), \\
& \bar{y}=g(\bar{x}, \bar{x}, \bar{x}, \bar{x}, \bar{y}, \bar{y}, \bar{y}, \bar{y}) .
\end{aligned}
$$

The point $(\bar{x}, \bar{y})$ is also called a fixed point of the vector map $F$.

Definition 1.1 Let $(\bar{x}, \bar{y})$ be an equilibrium point of the system (1.2).

(i) An equilibrium point $(\bar{x}, \bar{y})$ is said to be stable if for every $\varepsilon>0$, there exists $\delta>0$ such that for every initial condition $\left(x_{i}, y_{i}\right), i \in\{-3,-2,-1,0\}$ if $\left\|\sum_{i=-3}^{0}\left(x_{i}, y_{i}\right)-(\bar{x}, \bar{y})\right\|<\delta$ implies $\left\|\left(x_{n}, y_{n}\right)-(\bar{x}, \bar{y})\right\|<\varepsilon$ for all $n>0$, where $\|\cdot\|$ is the usual Euclidean norm in $\mathbb{R}^{2}$.

(ii) An equilibrium point $(\bar{x}, \bar{y})$ is said to be unstable if it is not stable.

(iii) An equilibrium point $(\bar{x}, \bar{y})$ is said to be asymptotically stable if there exists $\eta>0$ such that $\left\|\sum_{i=-3}^{0}\left(x_{i}, y_{i}\right)-(\bar{x}, \bar{y})\right\|<\eta$ and $\left(x_{n}, y_{n}\right) \rightarrow(\bar{x}, \bar{y})$ as $n \rightarrow \infty$.

(iv) An equilibrium point $(\bar{x}, \bar{y})$ is called a global attractor if $\left(x_{n}, y_{n}\right) \rightarrow(\bar{x}, \bar{y})$ as $n \rightarrow \infty$.

(v) An equilibrium point $(\bar{x}, \bar{y})$ is called an asymptotic global attractor if it is a global attractor and stable.

Definition 1.2 Let $(\bar{x}, \bar{y})$ be an equilibrium point of the map

$$
F=\left(f, x_{n}, x_{n-1}, x_{n-2}, x_{n-3}, g, y_{n}, y_{n-1}, y_{n-2}, y_{n-3}\right) \text {, }
$$

where $f$ and $g$ are continuously differentiable functions at $(\bar{x}, \bar{y})$. The linearized system of (1.2) about the equilibrium point $(\bar{x}, \bar{y})$ is

$$
X_{n+1}=F\left(X_{n}\right)=F_{J} X_{n},
$$


where

$$
X_{n}=\left(\begin{array}{c}
x_{n} \\
x_{n-1} \\
x_{n-2} \\
x_{n-3} \\
y_{n} \\
y_{n-1} \\
y_{n-2} \\
y_{n-3}
\end{array}\right)
$$

and $F_{J}$ is a Jacobian matrix of the system (1.2) about the equilibrium point $(\bar{x}, \bar{y})$.

To construct the corresponding linearized form of the system (1.1), we consider the following transformation:

$$
\left(x_{n}, x_{n-1}, x_{n-2}, x_{n-3}, y_{n}, y_{n-1}, y_{n-2}, y_{n-3}\right) \mapsto\left(f, f_{1}, f_{2}, f_{3}, f_{4}, g, g_{1}, g_{2}, g_{3}, g_{4}\right),
$$

where $f=\frac{\alpha x_{n-3}}{\beta+\gamma y_{n} y_{n-1} y_{n-2} y_{n-3}}, g=\frac{\alpha_{1} y_{n-3}}{\beta_{1}+\gamma_{1} x_{n} x_{n-1} x_{n-2} x_{n-3}}, f_{1}=x_{n}, f_{2}=x_{n-1}, f_{3}=x_{n-2}, f_{4}=x_{n-3}, g_{1}=y_{n}$, $g_{2}=y_{n-1}, g_{3}=y_{n-2}$ and $g_{4}=y_{n-3}$. The Jacobian matrix about the fixed point $(\bar{x}, \bar{y})$ under the transformation (1.3) is given by

$$
F_{J}(\bar{x}, \bar{y})=\left(\begin{array}{cccccccc}
0 & 0 & 0 & A & B & B & B & B \\
1 & 0 & 0 & 0 & 0 & 0 & 0 & 0 \\
0 & 1 & 0 & 0 & 0 & 0 & 0 & 0 \\
0 & 0 & 1 & 0 & 0 & 0 & 0 & 0 \\
C & C & C & C & 0 & 0 & 0 & D \\
0 & 0 & 0 & 0 & 1 & 0 & 0 & 0 \\
0 & 0 & 0 & 0 & 0 & 1 & 0 & 0 \\
0 & 0 & 0 & 0 & 0 & 0 & 1 & 0
\end{array}\right),
$$

where $A=\frac{\alpha}{\beta+\gamma \bar{y}^{4}}, B=-\frac{\alpha \gamma \bar{x} \bar{y}^{3}}{(\beta+\gamma+\bar{y})^{2}}, C=-\frac{\alpha_{1} \gamma_{1} \overline{x^{3}}}{\left(\beta_{1}+\gamma_{1} \bar{x}^{4}\right)^{2}}$ and $D=\frac{\alpha_{1}}{\beta_{1}+\gamma_{1} \bar{x}^{4}}$.

Theorem 1.3 For the system $X_{n+1}=F\left(X_{n}\right), n=0,1, \ldots$, of difference equations such that $\bar{X}$ is a fixed point of $F$. If all eigenvalues of the Jacobian matrix $J_{F}$ about $\bar{X}$ lie inside the open unit disk $|\lambda|<1$, then $\bar{X}$ is locally asymptotically stable. If one of them has a modulus greater than one, then $\bar{X}$ is unstable.

Theorem 1.4 (Routh-Hurwitz criterion)

For real numbers $a_{1}, a_{2}, \ldots, a_{n}$, let

$$
P(\lambda)=\lambda^{n}+a_{1} \lambda^{n-1}+\cdots+a_{n-1} \lambda+a_{n}
$$

Consider the polynomial equation

$$
P(\lambda)=0 .
$$


We define the $n$ matrices as follows:

$$
\begin{aligned}
H_{1} & =\left(a_{1}\right), \quad H_{2}=\left(\begin{array}{cc}
a_{1} & 1 \\
a_{3} & a_{2}
\end{array}\right), \quad H_{3}=\left(\begin{array}{lll}
a_{1} & 1 & 0 \\
a_{3} & a_{2} & a_{1} \\
a_{5} & a_{4} & a_{3}
\end{array}\right), \\
H_{j} & =\left(\begin{array}{ccccc}
a_{1} & 1 & 0 & \cdots & 0 \\
a_{3} & a_{2} & a_{1} & \cdots & 0 \\
a_{5} & a_{4} & a_{3} & \cdots & 0 \\
\cdots & \ldots & \cdots & \cdots & \cdots \\
a_{2 j-1} & a_{2 j-2} & a_{2 j-3} & \cdots & a_{j}
\end{array}\right),
\end{aligned}
$$

where $(l, m)$ element in the matrix $H_{j}$, for $0<2 l-m<k$ is

$$
\begin{aligned}
& a_{2 l-m}=\left\{\begin{array}{lll}
1 & \text { for } 2 l=m, \\
0 & \text { for } 2 l<m \text { or } 2 l>m+k,
\end{array}\right. \\
& H_{n}=\left(\begin{array}{ccccc}
a_{1} & 1 & 0 & \cdots & 0 \\
a_{3} & a_{2} & a_{1} & \cdots & 0 \\
a_{5} & a_{4} & a_{3} & \cdots & 0 \\
\cdots & \cdots & \cdots & \cdots & \cdots \\
0 & 0 & 0 & \cdots & a_{n}
\end{array}\right) .
\end{aligned}
$$

The following statements are true:

(i) A necessary and sufficient condition for all of the roots of (1.5) to have a negative real part is $\operatorname{det}\left(H_{j}\right)>0$ for $j=1,2, \ldots, n$.

(ii) A necessary and sufficient condition for the existence of a root of (1.5) with a positive real part is $\operatorname{det}\left(H_{j}\right)<0$ for some $j \in\{1,2, \ldots, n\}$.

\section{Main results}

Let $(\bar{x}, \bar{y})$ be an equilibrium point of the system (1.1), then for $\alpha>\beta$ and $\alpha_{1}>\beta_{1}$, the system (1.1) has the following five equilibrium points:

$$
P_{0}=(0,0), \quad P_{1}=(A, B), \quad P_{2}=(-A, B), \quad P_{3}=(A,-B), \quad P_{4}=(-A,-B),
$$

where $A=\left(\frac{\alpha_{1}-\beta_{1}}{\gamma_{1}}\right)^{\frac{1}{4}}$ and $B=\left(\frac{\alpha-\beta}{\gamma}\right)^{\frac{1}{4}}$.

Theorem 2.1 Let $\left(x_{n}, y_{n}\right)$ be a positive solution of the system (1.1), then for every $m \geq 0$, the following results hold:

$$
\begin{aligned}
& 0 \leq x_{n} \leq\left(\frac{\alpha}{\beta}\right)^{m+1} x_{-3}, \quad \text { if } n=4 m+1, \\
& 0 \leq x_{n} \leq\left(\frac{\alpha}{\beta}\right)^{m+1} x_{-2}, \quad \text { if } n=4 m+2, \\
& 0 \leq x_{n} \leq\left(\frac{\alpha}{\beta}\right)^{m+1} x_{-1}, \quad \text { if } n=4 m+3,
\end{aligned}
$$




$$
\begin{aligned}
& 0 \leq x_{n} \leq\left(\frac{\alpha}{\beta}\right)^{m+1} x_{0}, \quad \text { if } n=4 m+4, \\
& 0 \leq y_{n} \leq\left(\frac{\alpha_{1}}{\beta_{1}}\right)^{m+1} y_{-3}, \quad \text { if } n=4 m+1, \\
& 0 \leq y_{n} \leq\left(\frac{\alpha_{1}}{\beta_{1}}\right)^{m+1} y_{-2}, \quad \text { if } n=4 m+2, \\
& 0 \leq y_{n} \leq\left(\frac{\alpha_{1}}{\beta_{1}}\right)^{m+1} y_{-1}, \quad \text { if } n=4 m+3, \\
& 0 \leq y_{n} \leq\left(\frac{\alpha_{1}}{\beta_{1}}\right)^{m+1} y_{0}, \quad \text { if } n=4 m+4 .
\end{aligned}
$$

Proof The results are obviously true for $m=0$. Suppose that results are true for $m=k \geq 1$, i.e.,

$$
\begin{aligned}
& 0 \leq x_{n} \leq\left(\frac{\alpha}{\beta}\right)^{k+1} x_{-3}, \quad \text { if } n=4 k+1, \\
& 0 \leq x_{n} \leq\left(\frac{\alpha}{\beta}\right)^{k+1} x_{-2}, \quad \text { if } n=4 k+2, \\
& 0 \leq x_{n} \leq\left(\frac{\alpha}{\beta}\right)^{k+1} x_{-1}, \quad \text { if } n=4 k+3, \\
& 0 \leq x_{n} \leq\left(\frac{\alpha}{\beta}\right)^{k+1} x_{0}, \quad \text { if } n=4 k+4, \\
& 0 \leq y_{n} \leq\left(\frac{\alpha_{1}}{\beta_{1}}\right)^{k+1} y_{-3}, \quad \text { if } n=4 k+1, \\
& 0 \leq y_{n} \leq\left(\frac{\alpha_{1}}{\beta_{1}}\right)^{k+1} y_{-2}, \quad \text { if } n=4 k+2, \\
& 0 \leq y_{n} \leq\left(\frac{\alpha_{1}}{\beta_{1}}\right)^{k+1} y_{-1}, \quad \text { if } n=4 k+3, \\
& 0 \leq y_{n} \leq\left(\frac{\alpha_{1}}{\beta_{1}}\right)^{k+1} y_{0}, \quad \text { if } n=4 k+4 .
\end{aligned}
$$

Now, for $m=k+1$ using (1.1), one has

$$
\begin{aligned}
0 & \leq x_{4 k+5}=\frac{\alpha x_{4 k+1}}{\beta+\gamma y_{4 k+4} y_{4 k+3} y_{4 k+2} y_{4 k+1}} \\
& \leq \frac{\alpha x_{4 k+1}}{\beta} \leq\left(\frac{\alpha}{\beta}\right)^{k+2} x_{-3} \\
0 & \leq x_{4 k+6}=\frac{\alpha x_{4 k+2}}{\beta+\gamma y_{4 k+5} y_{4 k+4} y_{4 k+3} y_{4 k+2}} \\
& \leq \frac{\alpha x_{4 k+2}}{\beta} \leq\left(\frac{\alpha}{\beta}\right)^{k+2} x_{-2}, \\
0 & \leq x_{4 k+7}=\frac{\alpha x_{4 k+3}}{\beta+\gamma y_{4 k+6} y_{4 k+5} y_{4 k+4} y_{4 k+3}}
\end{aligned}
$$




$$
\begin{aligned}
& \leq \frac{\alpha x_{4 k+3}}{\beta} \leq\left(\frac{\alpha}{\beta}\right)^{k+2} x_{-1}, \\
& 0 \leq x_{4 k+8}=\frac{\alpha x_{4 k+4}}{\beta+\gamma y_{4 k+7} y_{4 k+6} y_{4 k+5} y_{4 k+4}} \\
& \leq \frac{\alpha x_{4 k+4}}{\beta} \leq\left(\frac{\alpha}{\beta}\right)^{k+2} x_{0} \text {, } \\
& 0 \leq y_{4 k+5}=\frac{\alpha_{1} y_{4 k+1}}{\beta_{1}+\gamma_{1} x_{4 k+4} x_{4 k+3} x_{4 k+2} x_{4 k+1}} \\
& \leq \frac{\alpha_{1} y_{4 k+1}}{\beta_{1}} \leq\left(\frac{\alpha_{1}}{\beta_{1}}\right)^{k+2} y_{-3} \text {, } \\
& 0 \leq y_{4 k+6}=\frac{\alpha_{1} y_{4 k+2}}{\beta_{1}+\gamma_{1} x_{4 k+5} x_{4 k+4} x_{4 k+3} x_{4 k+2}} \\
& \leq \frac{\alpha_{1} y_{4 k+2}}{\beta_{1}} \leq\left(\frac{\alpha_{1}}{\beta_{1}}\right)^{k+2} y_{-2} \text {, } \\
& 0 \leq y_{4 k+7}=\frac{\alpha_{1} y_{4 k+3}}{\beta_{1}+\gamma_{1} x_{4 k+6} x_{4 k+5} x_{4 k+4} x_{4 k+3}} \\
& \leq \frac{\alpha_{1} y_{4 k+3}}{\beta_{1}} \leq\left(\frac{\alpha_{1}}{\beta_{1}}\right)^{k+2} y_{-1} \text {, } \\
& 0 \leq y_{4 k+8}=\frac{\alpha_{1} y_{4 k+4}}{\beta_{1}+\gamma_{1} x_{4 k+7} x_{4 k+6} x_{4 k+5} x_{4 k+4}} \\
& \leq \frac{\alpha_{1} y_{4 k+4}}{\beta_{1}} \leq\left(\frac{\alpha_{1}}{\beta_{1}}\right)^{k+2} y_{0} \text {. }
\end{aligned}
$$

Theorem 2.2 For the equilibrium point $P_{0}=(0,0)$ of Equation (1.1), the following results hold:

(i) Let $\alpha<\beta$ and $\alpha_{1}<\beta_{1}$, then the equilibrium point $P_{0}=(0,0)$ of the system (1.1) is locally asymptotically stable.

(ii) If $\alpha>\beta$ or $\alpha_{1}>\beta_{1}$, then the equilibrium point $P_{0}=(0,0)$ of the system (1.1) is unstable.

Proof (i) The linearized system of $(1.1)$ about the equilibrium point $(0,0)$ is given by

$$
X_{n+1}=F_{J}(0,0) X_{n},
$$

where

$$
X_{n}=\left(\begin{array}{c}
x_{n} \\
x_{n-1} \\
x_{n-2} \\
x_{n-3} \\
y_{n} \\
y_{n-1} \\
y_{n-2} \\
y_{n-3}
\end{array}\right)
$$


and

$$
F_{J}(0,0)=\left(\begin{array}{cccccccc}
0 & 0 & 0 & \frac{\alpha}{\beta} & 0 & 0 & 0 & 0 \\
1 & 0 & 0 & 0 & 0 & 0 & 0 & 0 \\
0 & 1 & 0 & 0 & 0 & 0 & 0 & 0 \\
0 & 0 & 1 & 0 & 0 & 0 & 0 & 0 \\
0 & 0 & 0 & 0 & 0 & 0 & 0 & \frac{\alpha_{1}}{\beta_{1}} \\
0 & 0 & 0 & 0 & 1 & 0 & 0 & 0 \\
0 & 0 & 0 & 0 & 0 & 1 & 0 & 0 \\
0 & 0 & 0 & 0 & 0 & 0 & 1 & 0
\end{array}\right) .
$$

The characteristic polynomial of $F_{J}(0,0)$ is given by

$$
P(\lambda)=\lambda^{8}-\left(\frac{\alpha}{\beta}+\frac{\alpha_{1}}{\beta_{1}}\right) \lambda^{4}-\frac{\alpha \alpha_{1}}{\beta \beta_{1}} .
$$

The roots of $P(\lambda)$ are $\lambda= \pm \frac{\alpha}{\beta}, \lambda= \pm \frac{\alpha_{1}}{\beta_{1}}, \lambda= \pm \iota \frac{\alpha}{\beta}, \lambda= \pm \iota \frac{\alpha_{1}}{\beta_{1}}$. Since all eigenvalues of the Jacobian matrix $F_{J}(0,0)$ about $(0,0)$ lie in an open unit dick $|\lambda|<1$, the equilibrium point $(0,0)$ is locally asymptotically stable.

(ii) It is easy to see that if $\alpha>\beta$ or $\alpha_{1}>\beta_{1}$, then there exists at least one root $\lambda$ of Equation (2.1) such that $|\lambda|>1$. Hence, by Theorem 1.3 if $\alpha>\beta$ or $\alpha_{1}>\beta_{1}$, then $(0,0)$ is unstable.

Theorem 2.3 If $\alpha>\beta$ and $\alpha_{1}>\beta_{1}$, then a positive equilibrium point $P_{1}=\left(\left(\frac{\alpha_{1}-\beta_{1}}{\gamma_{1}}\right)^{\frac{1}{4}},\left(\frac{\alpha-\beta}{\gamma}\right)^{\frac{1}{4}}\right)$ of Equation (1.1) is unstable.

Proof The linearized system of (1.1) about the equilibrium point $P_{1}$ is given by

$$
X_{n+1}=F_{J}\left(P_{1}\right) X_{n}
$$

where

$$
X_{n}=\left(\begin{array}{c}
x_{n} \\
x_{n-1} \\
x_{n-2} \\
x_{n-3} \\
y_{n} \\
y_{n-1} \\
y_{n-2} \\
y_{n-3}
\end{array}\right)
$$


and

$$
F_{J}\left(P_{1}\right)=\left(\begin{array}{cccccccc}
0 & 0 & 0 & 1 & L & L & L & L \\
1 & 0 & 0 & 0 & 0 & 0 & 0 & 0 \\
0 & 1 & 0 & 0 & 0 & 0 & 0 & 0 \\
0 & 0 & 1 & 0 & 0 & 0 & 0 & 0 \\
M & M & M & M & 0 & 0 & 0 & 1 \\
0 & 0 & 0 & 0 & 1 & 0 & 0 & 0 \\
0 & 0 & 0 & 0 & 0 & 1 & 0 & 0 \\
0 & 0 & 0 & 0 & 0 & 0 & 1 & 0
\end{array}\right),
$$

where

$$
L=-\left(\frac{\gamma_{1}}{\gamma}\right)^{\frac{1}{4}} \frac{\left(\alpha_{1}-\beta_{1}\right)^{\frac{1}{4}(\alpha-\beta)^{\frac{3}{4}}}}{\alpha}
$$

and

$$
M=-\left(\frac{\gamma}{\gamma_{1}}\right)^{\frac{1}{4}} \frac{(\alpha-\beta)^{\frac{1}{4}\left(\alpha_{1}-\beta_{1}\right)^{\frac{3}{4}}}}{\alpha_{1}} .
$$

The characteristic polynomial of $F_{J}\left(P_{1}\right)$ is given by

$$
P(\lambda)=\lambda^{8}-L M \lambda^{6}-2 L M \lambda^{5}-3 L M \lambda^{4}-4 L M \lambda^{3}-3 L M \lambda^{2}-2 L M \lambda-L M+1 .
$$

The roots of the characteristic polynomial $P(\lambda)$ given in Equation (2.2) are given by

$$
-1, \quad \pm \iota, \quad 1 \pm \sqrt{L} \sqrt{M}
$$

It is sufficient to prove that any one of these roots has absolute value greater than one. For this, consider

$$
\begin{aligned}
|1-\sqrt{L} \sqrt{M}| & =\left|1+\sqrt{\left(\frac{\alpha-\beta}{\alpha}\right)\left(\frac{\alpha_{1}-\beta_{1}}{\alpha_{1}}\right)}\right| \\
& =\left|1+\sqrt{\left(1-\frac{\beta}{\alpha}\right)\left(1-\frac{\beta_{1}}{\alpha_{1}}\right)}\right|>1 .
\end{aligned}
$$

Hence, by Theorem 1.3 if $\alpha>\beta$ and $\alpha_{1}>\beta_{1}$, then $P_{1}$ is unstable.

Theorem 2.4 If $\alpha>\beta$ and $\alpha_{1}>\beta_{1}$, then the equilibrium points $P_{2}, P_{3}, P_{4}$ of Equation (1.1) are unstable.

Proof The proof is similar to Theorem 2.3, so it is omitted.

The following theorem is similar to Theorem 3.4 of [9].

Theorem 2.5 Let $\alpha>\beta$ and $\alpha_{1}>\beta_{1}$, and let $\left(x_{n}, y_{n}\right)$ be a solution of the system (1.1). Then, for $k=-3,-2,-1,0$, the following statements are true: 
(i) If $\left(x_{k}, y_{k}\right) \in\left(0,\left(\frac{\alpha_{1}-\beta_{1}}{\gamma_{1}}\right)^{\frac{1}{4}}\right) \times\left(\left(\frac{\alpha-\beta}{\gamma}\right)^{\frac{1}{4}}, \infty\right)$, then $\left(x_{n}, y_{n}\right) \in\left(0,\left(\frac{\alpha_{1}-\beta_{1}}{\gamma_{1}}\right)^{\frac{1}{4}}\right) \times\left(\left(\frac{\alpha-\beta}{\gamma}\right)^{\frac{1}{4}}, \infty\right)$.

(ii) If $\left(x_{k}, y_{k}\right) \in\left(\left(\frac{\alpha_{1}-\beta_{1}}{\gamma_{1}}\right)^{\frac{1}{4}}, \infty\right) \times\left(0,\left(\frac{\alpha-\beta}{\gamma}\right)^{\frac{1}{4}}\right)$, then $\left(x_{n}, y_{n}\right) \in\left(\left(\frac{\alpha_{1}-\beta_{1}}{\gamma_{1}}\right)^{\frac{1}{4}}, \infty\right) \times\left(0,\left(\frac{\alpha-\beta}{\gamma}\right)^{\frac{1}{4}}\right)$.

Theorem 2.6 The system (1.1) has no prime period-two solutions.

Proof Assume that $\left(p_{1}, q_{1}\right),\left(p_{2}, q_{2}\right),\left(p_{1}, q_{1}\right), \ldots$ is a prime period-two solution of Equation (1.1) such that $p_{i}, q_{i} \neq 0$ and $p_{i} \neq q_{i}$ for $i=1,2$. Then, from the system (1.1), one has

$$
p_{1}=\frac{\alpha p_{1}}{\beta+\gamma\left(q_{1} q_{2}\right)^{2}}, \quad p_{2}=\frac{\alpha p_{2}}{\beta+\gamma\left(q_{1} q_{2}\right)^{2}},
$$

and

$$
q_{1}=\frac{\alpha_{1} q_{1}}{\beta_{1}+\gamma_{1}\left(p_{1} p_{2}\right)^{2}}, \quad q_{2}=\frac{\alpha_{1} q_{2}}{\beta_{1}+\gamma_{1}\left(p_{1} p_{2}\right)^{2}} .
$$

From (2.3) and (2.4), one has $p_{i}, q_{i}=0$ for $i=1,2$. Which is a contradiction. Hence, the system (1.1) has no prime period-two solutions.

Theorem 2.7 Let $\alpha<\beta$ and $\alpha_{1}<\beta_{1}$, then the equilibrium point $P_{0}=(0,0)$ of Equation (1.1) is globally asymptotically stable.

Proof For $\alpha<\beta$ and $\alpha_{1}<\beta_{1}$, from Theorem 2.2, $(0,0)$ is locally asymptotically stable. From Theorem 2.1, it is easy to see that every positive solution $\left(x_{n}, y_{n}\right)$ is bounded, i.e., $0 \leq x_{n} \leq \mu$ and $0 \leq y_{n} \leq v$ for all $n=0,1,2, \ldots$, where $\mu=\max \left\{x_{-3}, x_{-2}, x_{-1}, x_{0}\right\}$ and $v=\max \left\{y_{-3}, y_{-2}, y_{-1}, y_{0}\right\}$. Now, it is sufficient to prove that $\left(x_{n}, y_{n}\right)$ is decreasing. From the system (1.1), one has

$$
\begin{aligned}
x_{n+1} & =\frac{\alpha x_{n-3}}{\beta+\gamma y_{n} y_{n-1} y_{n-2} y_{n-3}} \\
& \leq \frac{\alpha x_{n-3}}{\beta}<x_{n-3} .
\end{aligned}
$$

This implies that $x_{4 n+1}<x_{4 n-3}$ and $x_{4 n+5}<x_{4 n+1}$. Hence, the subsequences $\left\{x_{4 n+1}\right\},\left\{x_{4 n+2}\right\}$, $\left\{x_{4 n+3}\right\},\left\{x_{4 n+4}\right\}$ are decreasing, i.e., the sequence $\left\{x_{n}\right\}$ is decreasing. Similarly, one has

$$
\begin{aligned}
y_{n+1} & =\frac{\alpha_{1} x_{n-3}}{\beta_{1}+\gamma_{1} y_{n} y_{n-1} y_{n-2} y_{n-3}} \\
& \leq \frac{\alpha_{1} y_{n-3}}{\beta_{1}}<y_{n-3} .
\end{aligned}
$$

This implies that $y_{4 n+1}<y_{4 n-3}$ and $y_{4 n+5}<y_{4 n+1}$. Hence, the subsequences $\left\{y_{4 n+1}\right\},\left\{y_{4 n+2}\right\}$, $\left\{y_{4 n+3}\right\},\left\{y_{4 n+4}\right\}$ are decreasing, i.e., the sequence $\left\{y_{n}\right\}$ is decreasing. Hence, $\lim _{n \rightarrow \infty} x_{n}=$ $\lim _{n \rightarrow \infty} y_{n}=0$.

Theorem 2.8 Let $\alpha>\beta$ and $\alpha_{1}>\beta_{1}$. Then, for a solution $\left(x_{n}, y_{n}\right)$ of the system (1.1), the following statements are true:

(i) If $x_{n} \rightarrow 0$, then $y_{n} \rightarrow \infty$.

(ii) If $y_{n} \rightarrow 0$, then $x_{n} \rightarrow \infty$. 


\section{Examples}

In order to verify our theoretical results and to support our theoretical discussions, we consider several interesting numerical examples in this section. These examples represent different types of qualitative behavior of solutions to the system of nonlinear difference equations (1.1). All plots in this section are drawn with mathematica.

Example Consider the system (1.1) with initial conditions $x_{-3}=1.1, x_{-2}=2.6, x_{-1}=1.6$, $x_{0}=1.7, y_{-3}=1.5, y_{-2}=1.3, y_{-1}=2.5, y_{0}=0.5$. Moreover, choosing the parameters $\alpha=$ $0.01, \beta=0.011, \gamma=50, \alpha_{1}=0.03, \beta_{1}=0.031, \gamma_{1}=70$, the system (1.1) can be written as follows:

$$
x_{n+1}=\frac{0.01 x_{n-3}}{0.011+50 y_{n} y_{n-1} y_{n-2} y_{n-3}}, \quad y_{n+1}=\frac{0.03 y_{n-3}}{0.031+70 x_{n} x_{n-1} x_{n-2} x_{n-3}},
$$

$n=0,1, \ldots$, and with initial conditions $x_{-3}=1.1, x_{-2}=2.6, x_{-1}=1.6, x_{0}=1.7, y_{-3}=1.5$, $y_{-2}=1.3, y_{-1}=2.5, y_{0}=0.5$. The plot of the system (3.1) is shown in Figure 1 and its global attractor is shown in Figure 2.
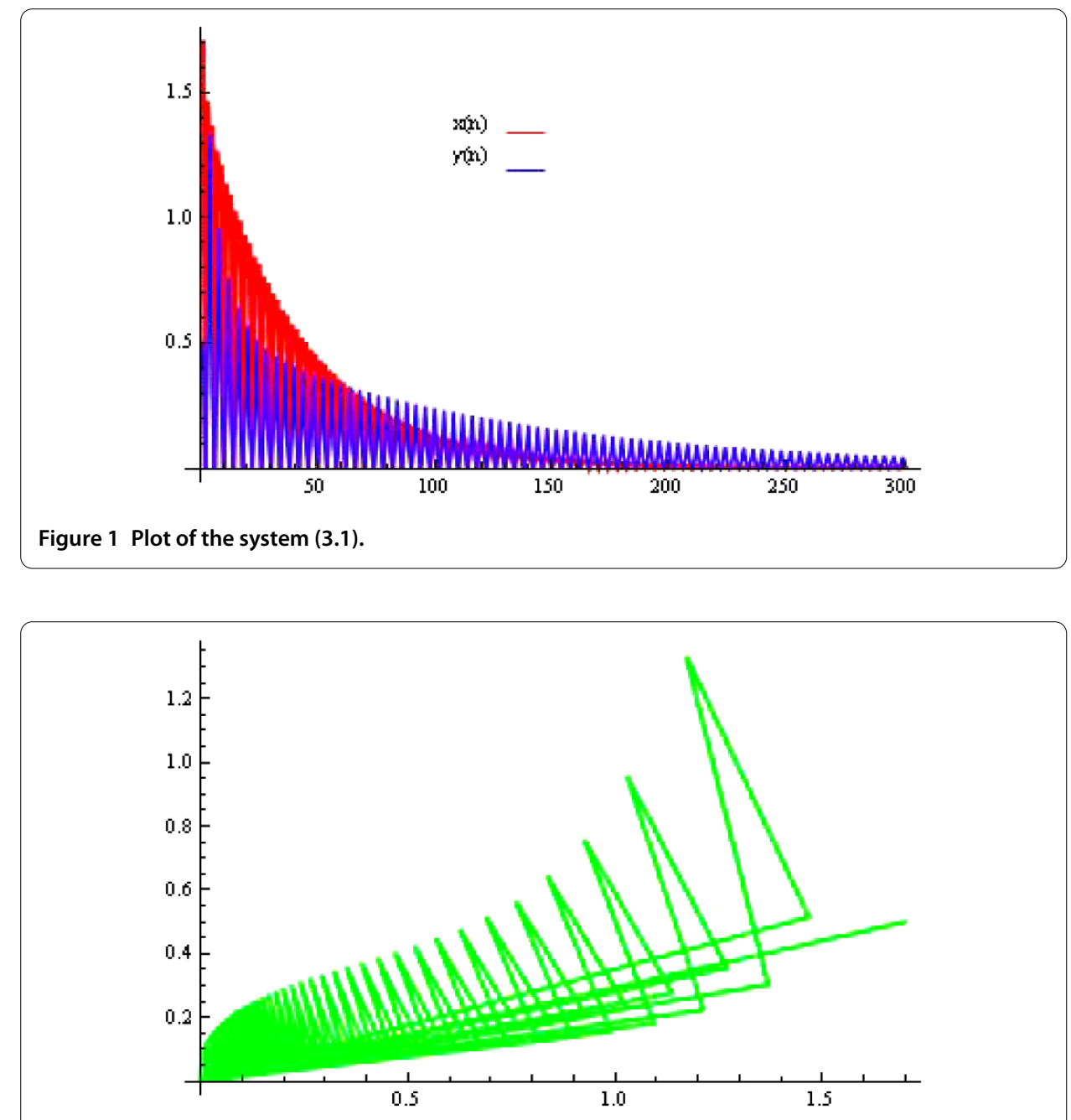

Figure 2 An attractor of the system (3.1). 


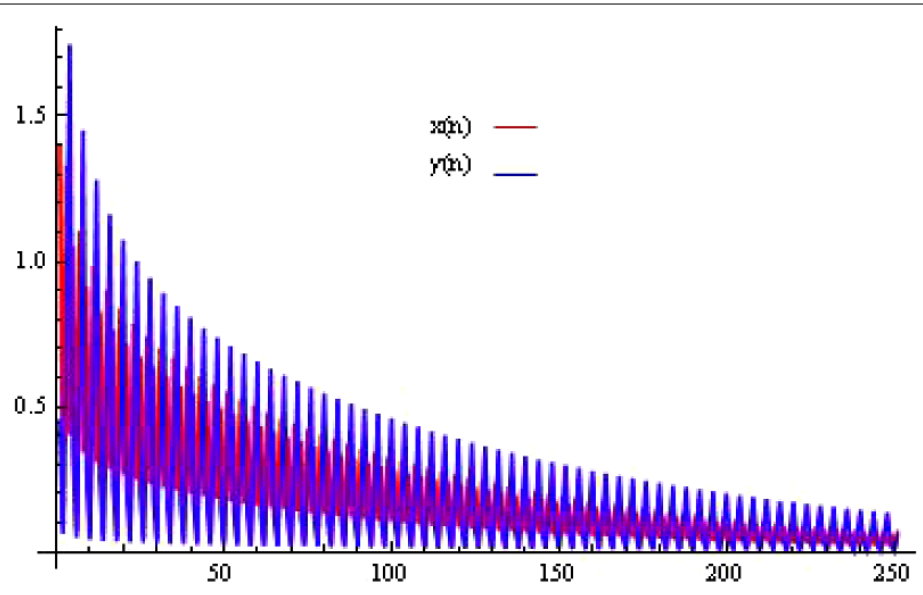

Figure 3 Plot of the system (3.1).

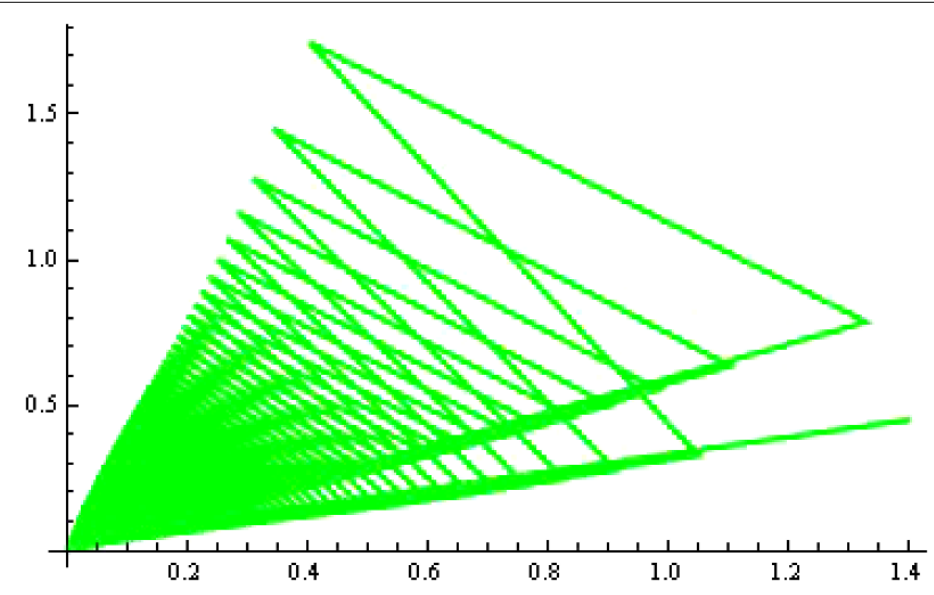

Figure 4 An attractor of the system (3.1).

Example Consider the system (1.1) with initial conditions $x_{-3}=2.1, x_{-2}=2.6, x_{-1}=0.6$, $x_{0}=1.4, y_{-3}=1.5, y_{-2}=1.6, y_{-1}=2.7, y_{0}=0.45$. Moreover, choosing the parameters $\alpha=12$, $\beta=12.5, \gamma=90, \alpha_{1}=15, \beta_{1}=15.5, \gamma_{1}=75$, the system (1.1) can be written as follows:

$$
x_{n+1}=\frac{12 x_{n-3}}{12.5+90 y_{n} y_{n-1} y_{n-2} y_{n-3}}, \quad y_{n+1}=\frac{15 y_{n-3}}{15.5+75 x_{n} x_{n-1} x_{n-2} x_{n-3}}
$$

$n=0,1, \ldots$, and with initial conditions $x_{-3}=2.1, x_{-2}=2.6, x_{-1}=0.6, x_{0}=1.4, y_{-3}=1.5$, $y_{-2}=1.6, y_{-1}=2.7, y_{0}=0.45$. The plot of the system (3.2) is shown in Figure 3 and its global attractor is shown in Figure 4.

Example Consider the system (1.1) with initial conditions $x_{-3}=9.2, x_{-2}=1.8, x_{-1}=0.76$, $x_{0}=1.1, y_{-3}=1.1, y_{-2}=1.2, y_{-1}=8.1, y_{0}=0.52$. Moreover, choosing the parameters $\alpha=$ 200, $\beta=225, \gamma=1000, \alpha_{1}=150, \beta_{1}=160, \gamma_{1}=700$, the system (1.1) can be written as follows:

$$
x_{n+1}=\frac{200 x_{n-3}}{225+1000 y_{n} y_{n-1} y_{n-2} y_{n-3}}, \quad y_{n+1}=\frac{150 y_{n-3}}{160+700 x_{n} x_{n-1} x_{n-2} x_{n-3}},
$$




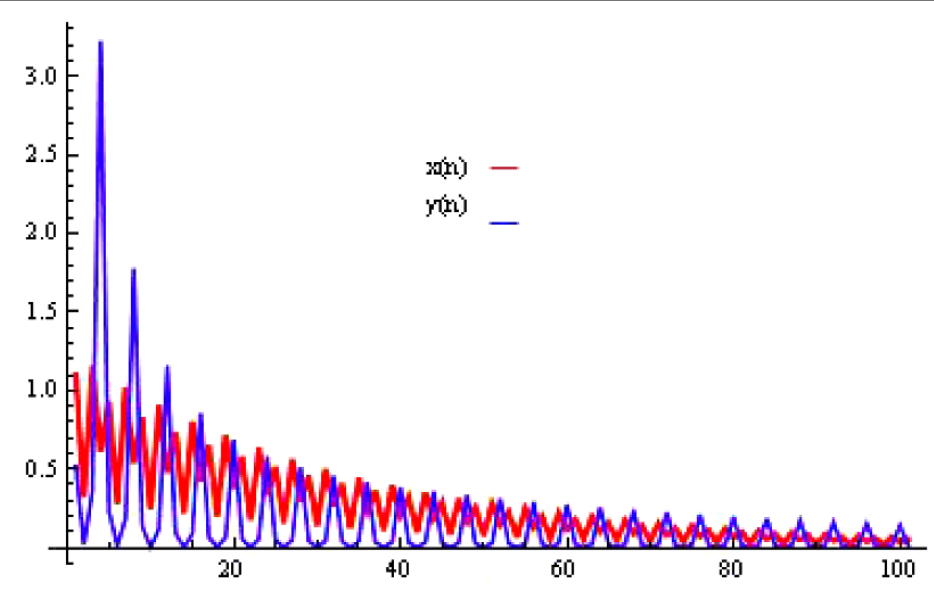

Figure 5 Plot of the system (3.3).

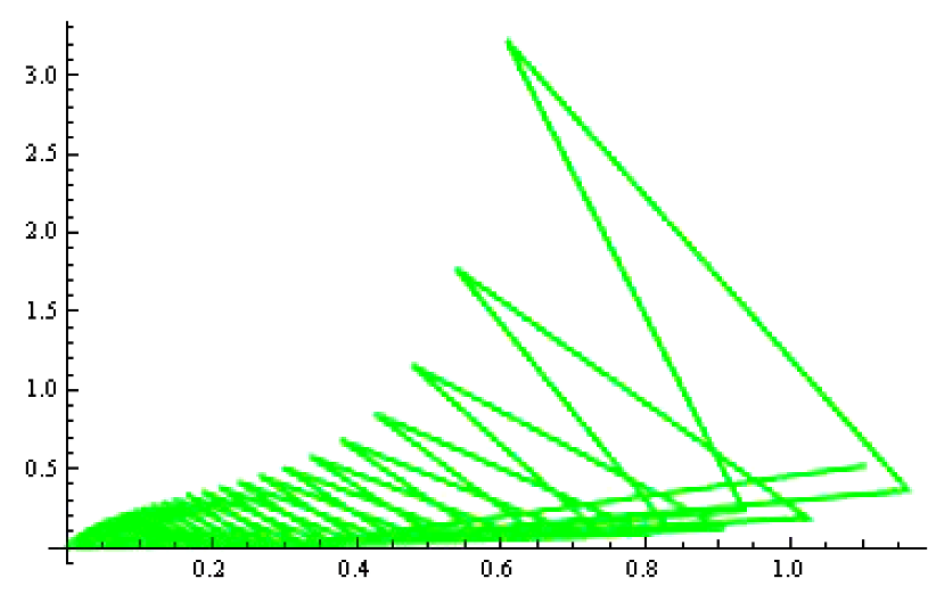

Figure 6 An attractor of the system (3.3).

$n=0,1, \ldots$, and with initial conditions $x_{-3}=9.2, x_{-2}=1.8, x_{-1}=0.76, x_{0}=1.1, y_{-3}=1.1$, $y_{-2}=1.2, y_{-1}=8.1, y_{0}=0.52$. The plot of the system (3.3) is shown in Figure 5 and its global attractor is shown in Figure 6.

Example Consider the system (1.1) with initial conditions $x_{-3}=1.3, x_{-2}=1.8, x_{-1}=2.6$, $x_{0}=2.1, y_{-3}=0.01, y_{-2}=1.2, y_{-1}=2.8, y_{0}=1.5$. Moreover, choosing the parameters $\alpha=12$, $\beta=10.5, \gamma=15, \alpha_{1}=14, \beta_{1}=13, \gamma_{1}=0.2$, the system (1.1) can be written as follows:

$$
x_{n+1}=\frac{12 x_{n-3}}{10.5+15 y_{n} y_{n-1} y_{n-2} y_{n-3}}, \quad y_{n+1}=\frac{14 y_{n-3}}{13+0.2 x_{n} x_{n-1} x_{n-2} x_{n-3}},
$$

$n=0,1, \ldots$, with initial conditions $x_{-3}=1.3, x_{-2}=1.8, x_{-1}=2.6, x_{0}=2.1, y_{-3}=0.01, y_{-2}=$ $1.2, y_{-1}=2.8, y_{0}=1.5$. The plot of the system (3.4) is shown in Figure 7 .

\section{Conclusion}

This work is a natural extension of $[9,10]$. In the paper, we investigated some dynamics of an eight-dimensional discrete system. The system has five equilibrium points all of 


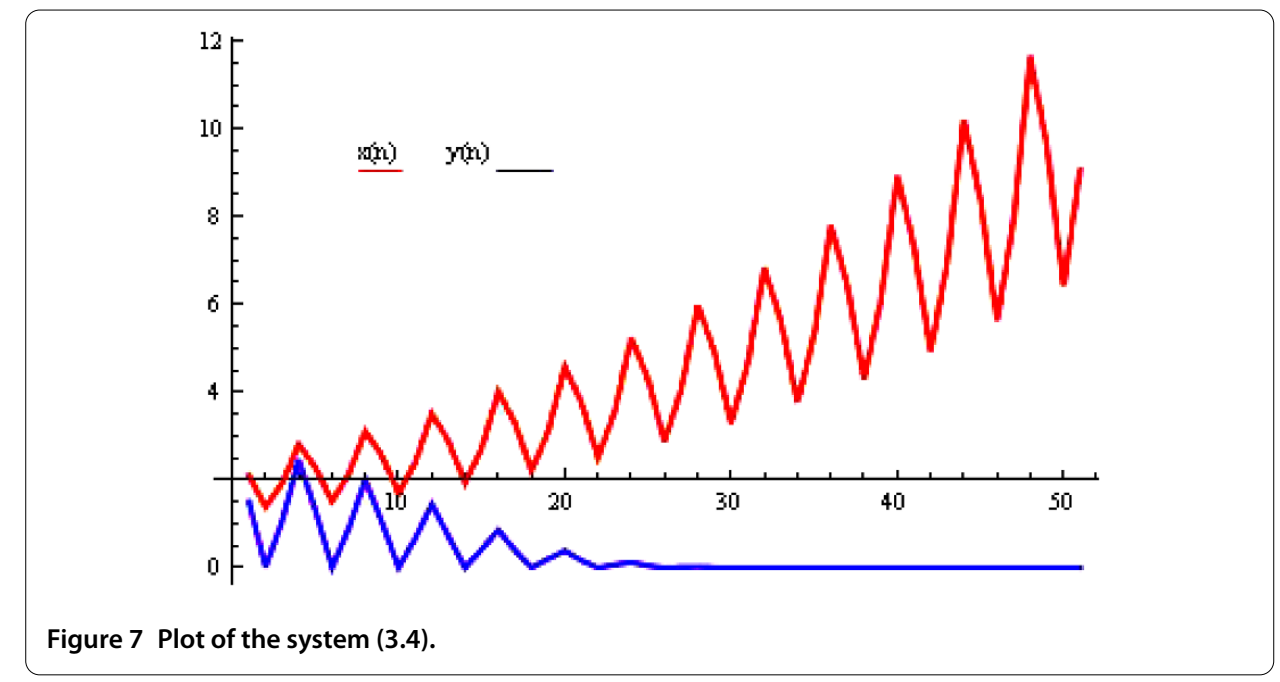

which except $(0,0)$ are unstable. The linearization method is used to show that the equilibrium point $(0,0)$ is locally asymptotically stable. We prove that the system has no prime period-two solutions. The main objective of dynamical systems theory is to predict the global behavior of a system based on the knowledge of its present state. An approach to this problem consists of determining the possible global behaviors of the system and determining which initial conditions lead to these long-term behaviors. In case of higher-order dynamical systems, it is crucial to discuss global behavior of the system. Some powerful tools such as semiconjugacy and weak contraction cannot be used to analyze global behavior of the system (1.1). In the paper, we prove the global asymptotic stability of the equilibrium point $(0,0)$ by using simple techniques. Some numerical examples are provided to support our theoretical results. These examples are experimental verifications of theoretical discussions.

Competing interests

The authors declare that they have no competing interests.

\section{Authors' contributions}

QD and MNQ carried out the theoretical proof and drafted the manuscript. AQK participated in the design and coordination. All authors read and approved the final manuscript.

\section{Acknowledgements \\ Authors would like to thank the referees for their comments and suggestions on the manuscript. This work was supported by the Higher Education Commission of Pakistan.}

Received: 3 October 2012 Accepted: 3 December 2012 Published: 17 December 2012

\section{References}

1. Cinar, C: On the positive solutions of the difference equation system $x_{n+1}=\frac{1}{y_{n}} ; y_{n+1}=\frac{y_{n}}{x_{n-1} y_{n-1}}$. Appl. Math. Comput. 158, 303-305 (2004)

2. Stević, S: On some solvable systems of difference equations. Appl. Math. Comput. 218, 5010-5018 (2012)

3. Kurbanli, AS: On the behavior of positive solutions of the system of rational difference equations $x_{n+1}=\frac{x_{n-1}}{y_{n} x_{n-1}-1}$, $y_{n+1}=\frac{y_{n-1}}{x_{n} y_{n-1}-1}, z_{n+1}=\frac{1}{y_{n} z_{n}}$. Adv. Differ. Equ. 2011, 40 (2011)

4. Bajo, I, Liz, E: Global behaviour of a second-order nonlinear difference equation. J. Differ. Equ. Appl. 17(10), 1471-1486 (2011)

5. Kalabusisić, S, Kulenović, MRS, Pilav, E: Dynamics of a two-dimensional system of rational difference equations of Leslie-Gower type. Adv. Differ. Equ. (2011). doi:10.1186/1687-1847-2011-29

6. Kurbanli, AS, Çinar, C, Yalçinkaya, I: On the behavior of positive solutions of the system of rational difference equations $x_{n+1}=\frac{x_{n-1}}{y_{n} x_{n-1}+1}, y_{n+1}=\frac{y_{n-1}}{x_{n} y_{n-1}+1}$. Math. Comput. Model. 53, 1261-1267 (2011)

7. Touafek, N, Elsayed, EM: On the solutions of systems of rational difference equations. Math. Comput. Model. 55, 1987-1997 (2012) 
8. Touafek, N, Elsayed, EM: On the periodicity of some systems of nonlinear difference equations. Bull. Math. Soc. Sci. Math. Roumanie 2, 217-224 (2012)

9. Zhang, Q, Yang, L, Liu, J: Dynamics of a system of rational third order difference equation. Adv. Differ. Equ. (2012). doi:10.1186/1687-1847-2012-136

10. Shojaei, M, Saadati, R, Adibi, H: Stability and periodic character of a rational third order difference equation. Chaos Solitons Fractals 39, 1203-1209 (2009)

doi:10.1186/1687-1847-2012-215

Cite this article as: Din et al.: Dynamics of a fourth-order system of rational difference equations. Advances in Difference Equations 2012 2012:215.

Submit your manuscript to a SpringerOpen ${ }^{\circ}$ journal and benefit from:

- Convenient online submission

- Rigorous peer review

- Immediate publication on acceptance

- Open access: articles freely available online

- High visibility within the field

- Retaining the copyright to your article 